\title{
Regression of Tumor Thrombus in the Suprahepatic Vena Cava of Hepatocellular Carcinoma and Conversion Hepatectomy Induced by Lenvatinib
}

\author{
Ryota Matsuki $^{\mathrm{a}}$ Naohiro Okano $^{\mathrm{b}}$ Takaaki Arai $^{\mathrm{a}}$ Shinya Yoshiike ${ }^{\mathrm{c}}$ \\ Masaharu Kogure $^{a}$ Yutaka Suzuki $^{a}$ Junji Shibahara ${ }^{c}$ Junji Furuse ${ }^{b}$ \\ Yoshihiro Sakamoto ${ }^{a}$ \\ aDepartment of Hepato-Biliary-Pancreatic Surgery Division, Kyorin University Hospital, Tokyo, Japan; \\ bDepartment of Medical Oncology, Kyorin University, Faculty of Medicine, Tokyo, Japan; \\ 'Department of Pathology, Kyorin University, Faculty of Medicine, Tokyo, Japan
}

Dear Editor,

Recent developments in systemic therapy for hepatocellular carcinoma (HCC) have been outstanding, and a response rate of $40.6 \%$ based on the modified RECIST criteria has been reported after lenvatinib (LEN) therapy for advanced HCC [1]. However, reports on conversion surgery (CS) after LEN are scarce [2]. A case of advanced HCC with tumor thrombus (TT) in the suprahepatic vena cava (SHVC) close to the right atrium, which shrank after 12 weeks' administration of LEN, thereby leading to successful CS without using total vascular exclusion (TVE) or extracorporeal circulation (EC), is presented.

The patient was an octogenarian male with alcoholic hepatopathy. Dynamic contrast-enhanced computed tomography showed a hypervascular mass in the right liver, $13 \mathrm{~cm}$ in diameter, with a TT in the SHVC through the right hepatic vein (RHV) (Fig. 1a). Because the tip of the TT was located at the level of the diaphragm, removal of the TT with the right hemiliver would require TVE of the inferior vena cava (IVC), possibly with TVE technique and median sternotomy. With a diagnosis of unresectable HCC, LEN was started at an initial dose of $8 \mathrm{mg} /$ day (pa-

karger@karger.com www.karger.com/lic

Karger:
(C) 2021 The Author(s)

Published by S. Karger AG, Basel

This is an Open Access article licensed under the Creative Commons Attribution-NonCommercial-4.0 International License (CC BY-NC) (http://www.karger.com/Services/OpenAccessLicense), applicable to the online version of the article only. Usage and distribution for commercial purposes requires written permission. tient's weight was $50 \mathrm{~kg}$ ) after obtaining informed consent on risk and benefits of LEN and alternative treatments for this stage of HCC. Four weeks after starting LEN, the tumor decreased by $15.6 \%$, suggesting stable disease on RECIST 1.1, and by $35.9 \%$, suggesting partial response on the modified RECIST. The tip of the TT withdrew from the SHVC to the orifice of the RHV (Fig. 1b). Thus, conversion right hemihepatectomy with removal of the TT was planned. Liver function was moderately deteriorated (ICG-R15 value of 13.1\%) and the future liver remnant volume was estimated to be $42.4 \%$ of total liver volume. Four weeks administration of LEN further decreased the functional reserve, and ICG-R15 value increased to $18.5 \%$. Preoperative right portal vein embolization (PVE) was performed, which increased the future liver remnant volume to 53.9\%. With continued treatment with LEN before and after PVE, the TT shrank further (Fig. 1c). Liver function improved after 2 weeks of discontinuation of LEN and ICG-R15 value decreased to $13.7 \%$. Thereafter, CS was performed following 12 weeks administration of LEN. The tip of the TT was located around the orifice of the RHV on intraoperative ultrasonography (Fig. 1d). At the 

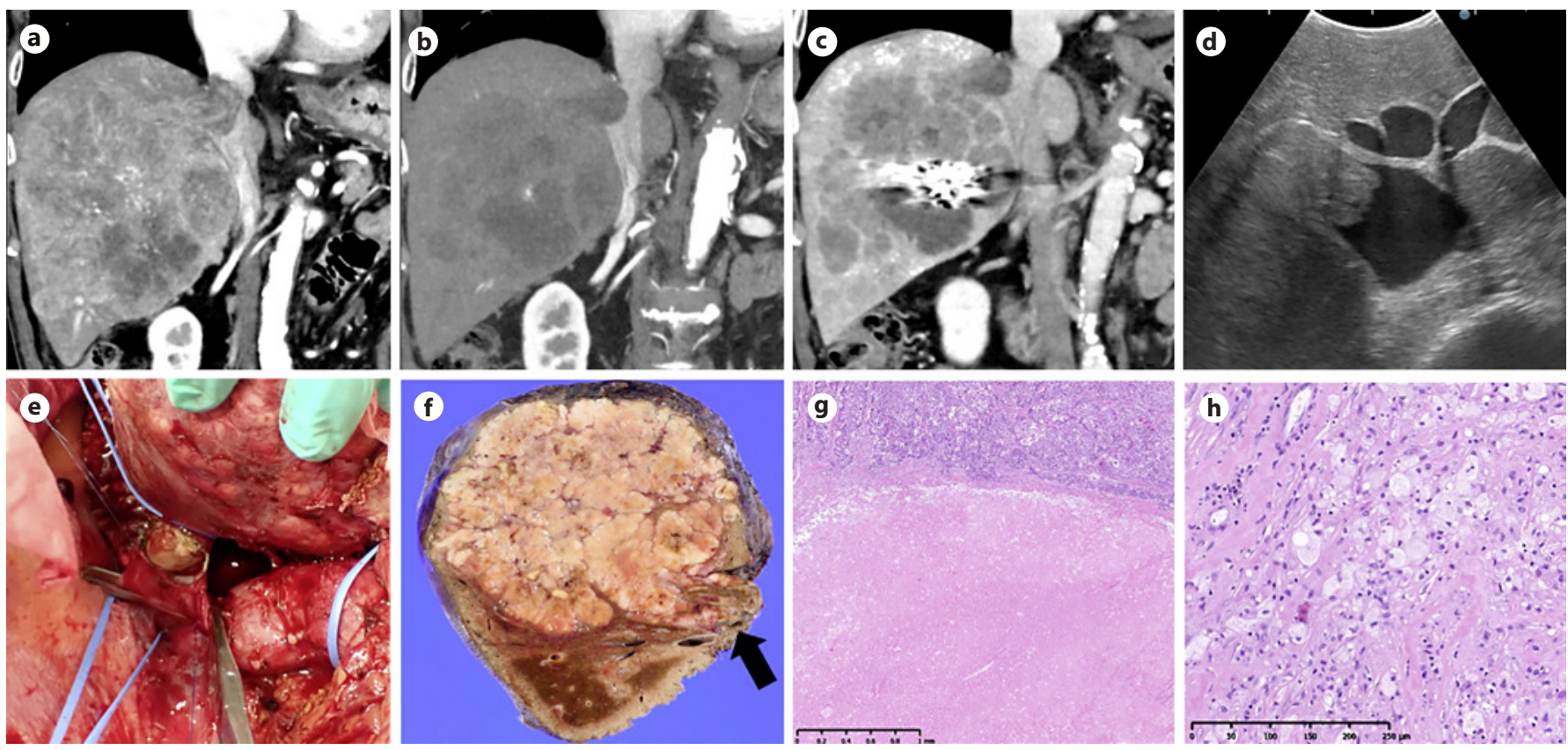

Fig. 1. a Retreatment dynamic CT. The hypervascular hepatic mass in the right liver with a tumor thrombus in the superior vena cava through the right hepatic vein, and the tip of the tumor thrombus was located at the level of the diaphragm. b Dynamic CT on 4 weeks after administration of LEN. The tip of the tumor thrombus withdrew from the superior vena cava to the orifice of the right hepatic vein. c Preoperative dynamic CT (11 weeks after administration of LEN). The tumor thrombus shrank further. d Intraoperative ultrasonography showed that the tip of the tumor thrombus was located around the orifice of the right hepatic vein. e The

last step of the hepatic transection, the wall of the RHV was opened after partial clamping of the IVC, and the IVCTT was extracted without difficulty (Fig. 1e). The operation time was $7 \mathrm{~h}$, and the estimated blood loss was 460 $\mathrm{mL}$. Macroscopically findings showed confluent multinodular type of the tumor, $15 \mathrm{~cm}$ in diameter, with hepatic vein tumor thrombus (HVTT) in the orifice of the RHV (Fig. 1f). Histopathological examination revealed poorly differentiated HCC. In hepatic tumor and TT, part of the tumor turned necrotic, and accumulation of foamy histocytes and fibrotic change were also shown, which suggested therapeutic response after chemotherapy (Fig. 1g, h). The patient was discharged on day 12 without severe complications. The patient is doing well without any sign of recurrence 4 months after surgery without any adjuvant chemotherapy.

The treatment strategy for HCC with macroscopic HVTT is controversial. In the Barcelona clinic liver cancer staging system, HCC with macro vascular invasion recommends systemic therapy not surgical resection

last step of the liver transection. The wall of the right hepatic vein was opened after partial clamping of the IVC, and the tumor thrombus was extracted from the IVC. $\mathbf{f}$ Macroscopical findings showed confluent multinodular type of the tumor, $15 \mathrm{~cm}$ in diameter, with hepatic vein tumor thrombus in the orifice of the right hepatic vein (Arrow). $\mathbf{g}, \mathbf{h}$ In hepatic tumor and TT, some of the tumor cells turned necrotic, and others showed accumulation of foamy histocytes and fibrosis, which suggested therapeutic response after chemotherapy. CT, computed tomography; TT, tumor thrombus; LEN, lenvatinib; IVC, inferior vena cava.

(SR). On the other hand, Kokudo et al. [3] reported that median survival times were similar between patients with TT in the peripheral HV and patients with TT in the major HV (4.85 vs. 4.67 years, $p=0.974$ ), and SR has been accepted as one of the treatment options for advanced HCC with HVTT in Japan. A retrospective cohort study reported by Wang et al. [4] showed that patients with SR survived significantly longer than patients with transcatheter arterial chemoembolization or systemic therapy for advanced HCC extending into the IVC or right atrium (median survival time: 19 months, 4.5 months, and 5.0 months, respectively). In the last 3 decades, resection of the TT in the SHVC or in the atrium from HCC was sporadically performed using $\mathrm{EC}$, but the 3 -year survival rate after resection of HCC having IVCTT with and without EC was reported to be $0 \%$ and $28 \%$, and some preoperative down-sizing therapy for IVCTT was advocated [5]. This is the first case of CS for advanced HCC with IVCTT. Safe removal of IVCTT without TVE, median sternotomy, or EC was performed after preoperative therapy with 
LEN. Preoperative LEN therapy might be a promising option among the multidisciplinary treatments for HCC with macroscopic HVTT.

\section{Acknowledgment}

We would like to give special thanks to Eisai for the support of investigator initiated studies.

\section{Conflicts of Interest Statement}

Junji Furuse: received honoraria from Eisai and is an editorial board member of Liver Cancer. Naohiro Okano received honoraria from Eisai. Other authors declare that they have no conflicts of interest or sources of funding for this manuscript.

\section{Funding Sources}

Junji Furuse received research fund from Eisai.

\section{Author Contributions}

N.O. and J.F. contributed to systemic lenvatinib therapy. R.M., T.A., Y.S., M.K., and Y. Sakamoto contributed to operation and perioperative management. J.S. and S.Y. contributed to histopathological examination. R.M. wrote the manuscript. All authors have seen and approved the final version of the manuscript being submitted, and all authors fulfill the COPE (Committee on Publication Ethics) requirements for authorship.

\section{References}

1 Kudo M, Finn RS, Qin S, Han KH, Ikeda K, Piscaglia F, et al. Lenvatinib versus sorafenib in first-line treatment of patients with unresectable hepatocellular carcinoma: a randomised phase 3 non-inferiority trial. Lancet. 2018 Mar 24;391(10126):1163-73.

2 Matsuki R, Kawai K, Suzuki Y, Kogure M, Nakazato T, Naruge D, et al. Pathological complete response in conversion hepatectomy induced by lenvatinib for advanced hepatocellular carcinoma. Liver Cancer. 2020 Jun;9(3): 358-60.
3 Kokudo T, Hasegawa K, Matsuyama Y, Takayama T, Izumi N, Kadoya M, et al. Liver resection for hepatocellular carcinoma associated with hepatic vein invasion: a Japanese nationwide survey. Hepatology. 2017 Aug; 66(2):510-7.

4 Wang Y, Yuan L, Ge RL, Sun Y, Wei G. Survival benefit of surgical treatment for hepatocellular carcinoma with inferior vena cava/ right atrium tumor thrombus: results of a retrospective cohort study. Ann Surg Oncol. 2013 Mar;20(3):914-22.
5 Kasai Y, Hatano E, Seo S, Taura K, Yasuchika $\mathrm{K}$, Okajima H, et al. Proposal of selection criteria for operative resection of hepatocellular carcinoma with inferior vena cava tumor thrombus incorporating hepatic arterial infusion chemotherapy. Surgery. 2017 Oct;162(4): $742-51$. 